\title{
EFICIÊNCIA TÉCNICA NA INDÚSTRIA DE BASE FLORESTAL BRASILEIRA VIA METAS INTERMEDIÁRIAS ${ }^{1}$
}

\author{
Ricardo Bruno Nascimento dos Santos²
}

\begin{abstract}
RESUMO - Propôs-se, neste artigo, uma busca de alvos, por etapas, com alvos intermediários nas camadas de isoeficiência para que o Setor de Fabricação de Produtos de Base Florestal (SFPBF) de cada Estado atinja, de forma gradual, nível de eficiência técnica mais elevado. Para isso, utilizaram-se dados da Pesquisa Industrial Anual (PIA) de 2007, do IBGE. Aplicou-se a Análise Envoltória de Dados (DEA) orientada ao insumo, tendo a receita líquida do setor como produto e como insumos o total do custo das operações industriais (COI) e as remunerações do setor (SRO). Dos 14 Estados analisados, o Espírito Santo foi o mais eficiente e o Rio de Janeiro, o mais ineficiente. Outros 12 Estados foram tecnicamente ineficientes, de forma que três devem, no curto prazo, buscar a camada 1 , três a camada 2, dois a camada 3 e três a camada 4 . Verificou-se que no Brasil o COI é a principal causa da ineficiência técnica.
\end{abstract}

Palavras-chave: Análise Envoltória de Dados, Isoeficiência e Indústria de transformação.

\section{TECHNICAL EFFICIENCY IN BRAZILIAN FOREST INDUSTRY BASE VIA MILESTONES}

\begin{abstract}
This work aimed to search for targets in stages with intermediate targets in the isoefficiency layers so that the Manufacturing Sector of Forest-Based Products (MSFBP) of each state gradually reached a higher technical level of efficiency. Data from Pesquisa Industrial Anual (PIA) (the Annual Industrial Survey) 2007 from Instituto Brasileiro de Geografia e Estatística - IBGE (Brazilian Institute of Geography and Statistics) were used. Data envelopment analysis (DEA) was applied geared towards inputs. Net revenue in the sector as a product and inputs was the total industrial operations cost (IOC) and wages in the sector (WS). Among fourteen analyzed states, Espírito Santo State is the most efficient and Rio de Janeiro the most inefficient. Another twelve states are technically inefficient, so that three states shall, in the short term, seek the layer 1, three for the layer 2, two for the layer 3, and three for the layer 4. It was found that in Brazil the IOC is the main cause of technical inefficiency.
\end{abstract}

Keywords: Data Envelopment Analysis, Isoefficiency and Manufacturing industry.

\section{INTRODUÇÃO}

Entre os setores da economia brasileira, o industrial foi responsável por $27 \%$ do Produto Interno Bruto (PIB) de 2007, mostrando grande importância para a geração de empregos e para o valor da produção, especialmente nas Regiões Nordeste, Sudeste e Sul (IBGE, 2007).

O setor industrial pode ser dividido em dois grandes grupos, segundo a sua atividade: indústria extrativa e indústria de transformação que geraram, respectivamente, 0,8; e 26,2\% do PIB de 2007. O Setor de Fabricação de Produtos de Base Florestal ${ }^{3}$ (SFPBF) está inserido na indústria de transformação e tem importante participação na economia, tendo contribuído com 9,43\% do valor da produção industrial de 2007 e com 15,22\% dos empregos da indústria de transformação nesse mesmo ano (IBGE, 2007).

O SFPBF corresponde ao último elo de várias cadeias produtivas do setor florestal, outro importante setor da economia brasileira, sendo responsável pela maior agregação de valor às matérias-primas de base florestal. Nesse setor, segundo Santana (2002), as

\footnotetext{
${ }^{1}$ Recebido em 24.04.2010 e aceito para publicação em 31.10.2011.

${ }^{2}$ Universidade Federal de Viçosa, UFV, Brasil. E-mail: <ricardobns@gmail.com>.
} 
firmas caracterizam-se pelo alto grau de rivalidade, e a necessidade de aumento da eficiência dos fatores de produção, como meio de aumentar a competitividade, é crucial, especialmente quando esse setor apresenta grande importância na geração de emprego e renda. Ademais, para Sampaio et al. (2005), com o aumento da eficiência há a possibilidade de redução na quantidade dos insumos utilizados, cujo excedente pode ser deslocado para uma nova esfera produtiva. Promover a melhora da eficiência de uma indústria pode diminuir a necessidade de expansão, aumentando os investimentos em outros setores do sistema produtivo.

A análise da eficiência técnica permite identificar quanto dos insumos pode ser reduzido, mantendo-se a produção constante, ou quanto a produção deve aumentar, mantendo-se fixa a quantidade de insumos utilizados para que uma unidade produtiva seja considerada eficiente no conjunto analisado, estabelecendo-se, assim, metas (alvos) a serem atingidas por cada unidade considerada ineficiente. Dessa forma, estabelecer metas a uma unidade produtiva significa definir diretrizes para sua produção ou utilização de insumos, de sorte que tais metas, se atingidas, levam a unidade analisada à fronteira eficiente. Como o SFPBF pode permitir grande agregação de valor à produção florestal e, portanto, à geração de renda, a análise da eficiência técnica com a estipulação de alvos torna-se importante, notadamente aqueles Estados onde o setor florestal apresenta grande participação em sua economia.

Podem ser encontradas, entretanto, barreiras práticas na realização em curto prazo dos alvos calculados. Assim, propôs-se, neste artigo, uma busca de alvos, por etapas, com alvos intermediários nas camadas de isoeficiência ${ }^{4}$ para que o SFPBF de cada Estado atinja nível de eficiência mais elevado. Faz-se, também, uma análise em termos dos tipos de retornos à escala presentes no SFPBF de cada unidade federativa analisada.

\section{MATERIAL E MÉTODOS}

\subsection{Referencial teórico}

A análise da eficiência técnica é baseada na teoria da produção, em que se define o conjunto de produção ${ }^{(}{ }^{\ominus}$ ), cujos elementos são todas as combinações de insumos e produtos que compreendem as formas tecnologicamente viáveis de produzir (VARIAN, 2000).

Supondo um vetor de insumos, $x \in \mathfrak{R}^{n}$, e um vetor de produtos, $x \in \mathfrak{R}^{m}$, diz-se que, quando $(\chi, \gamma)$ $\in{ }^{\Theta}$, esta é uma combinação tecnologicamente viável. O conjunto de produção mostra, portanto, as escolhas possíveis com as quais a empresa se defronta (SIMONSEN, 1993).

A eficiência técnica, entretanto, exige que se obtenha o máximo de produto possível de qualquer vetor de insumos. Essa é a fronteira do conjunto de produção, também chamada de fronteira ou função de produção $\left({ }^{\Phi}\right)$, que indica a quantidade máxima de produto que pode ser obtida a partir de determinado vetor de insumos, $x$.

Assim, é tecnicamente eficiente uma firma cuja combinação de insumos e produtos é tal que $(\chi, \gamma)$ $\in^{\Phi}$; caso contrário (se $(\chi, \gamma) \in \in^{\Phi}$ ), ela é considerada ineficiente, podendo ter obtido um vetor $\gamma^{\prime}>\gamma$ com o mesmo vetor $x$ ou, de outra forma, ter obtido $y$ com um vetor $\chi^{\prime}<\chi$.

\subsection{Modelo analítico}

De acordo com Sampaio et al. (2005), têm-se duas abordagens distintas para a medida de eficiência de unidades produtivas: a paramétrica e a não paramétrica. No primeiro caso, postula-se que a fronteira do conjunto produtivo pode ser representada por uma função de produção cuja forma funcional é definida a priori para a tecnologia. A especificação da função de produção é a maior limitação da abordagem paramétrica. As medidas de eficiência podem variar muito segundo a função

\footnotetext{
${ }^{3}$ O SFPBF é constituído pelas indústrias de Fabricação de produtos da madeira; Fabricação de celulose, papel e produtos de papel; Fabricação de artigos da borracha e material plástico; e Fabricação de móveis e indústrias diversas, porém, neste artigo foram trabalhadas apenas as indústrias de Fabricação de produtos da madeira e Fabricação de celulose, papel e produtos de papel por serem setores estritamente ligados ao setor florestal. As duas indústrias representam em média mais de $40 \%$ do número de empregados do SFPBF e são responsáveis por mais de 60\% da Receita Líquida de Vendas.

${ }^{4}$ Camadas de isoeficiência são fronteiras de produção, que podem se localizar abaixo ou sobre a fronteira eficiente, onde se assume que todas as DMU's que compõem cada camada apresentam o mesmo grau de eficiência técnica.
}

Revista Árvore, Viçosa-MG, v.35, n.6, p.1319-1326, 2011 
escolhida, sendo essa a diferença principal entre as duas abordagens. A não paramétrica não se baseia em uma função especificada a priori. A forma da fronteira do conjunto produtivo é determinada considerandose que o conjunto de produção deve satisfazer determinadas propriedades, não havendo suposições sobre a fronteira propriamente dita.

A Análise Envoltória de Dados (DEA) é um método não paramétrico de estimação de fronteira de produção para análise da eficiência relativa de um conjunto de entidades (chamadas de unidades tomadoras de decisão - DMU) na transformação de insumos em produtos. A DEA proporciona informações sobre a eficiência e classifica as DMUs como eficientes ou ineficientes, dependendo de sua posição geométrica em relação à fronteira eficiente gerada (DULÁ, 2002). A comparação é estritamente em relação aos membros do grupo estudado, e tradicionalmente considera-se que o número de DMUs deve ser, pelo menos, o triplo do número de variáveis para que seja obtida uma boa classificação (LETA et al., 2005).

De acordo com Becker et al. (2003), as principais vantagens da DEA são: a flexibilidade, uma vez que é uma técnica não paramétrica e não exige uma forma explícita de relacionamento entre insumos e produtos, sendo desnecessário o conhecimento detalhado do processo de produção; e a geração de cenários, identificando as DMUs eficientes e as ineficientes e também as DMUs eficientes que devem ser utilizadas como referência nas demais unidades (benchmarks).

Para Gomes et al. (2005), há dois modelos clássicos em DEA: o RCE (Retornos Constantes à Escala, também conhecido como CCR), inicialmente proposto por Charnes et al. (1978), que assumem proporcionalidade entre insumos e produtos; e o RVE (Retornos Variáveis à Escala, também conhecido como BCC), que substitui o axioma da proporcionalidade pelo da convexidade ${ }^{5}$. Além disso, são possíveis duas orientações: orientação aos insumos, quando se deseja minimizar a utilização de recursos, sem alteração do nível de produção; e orientação aos produtos, quando o objetivo é aumentar a produção, mantendo-se os insumos inalterados.
O modelo DEA-RVE pode ser representado pela seguinte notação algébrica (GOMES; BAPTISTA, 2004):

$$
\begin{aligned}
& \operatorname{MIN}_{\theta, \lambda} \theta \\
& \text { sujeito } a: \\
& -\gamma_{\mathrm{i}}+Y \lambda \geq 0 \\
& \theta \chi_{\mathrm{i}}-X \lambda \geq 0 \\
& N_{\mathrm{i}} \lambda=1 \\
& \lambda \geq 1
\end{aligned}
$$

em que $y$ é o produto da DMU sob análise; $x$ é o insumo da DMU sob análise; $X$ é a matriz de insumos $K \mathrm{x} N ; Y$ é a matriz de produtos $M$ x $N$; $\lambda$ é o vetor de constantes que multiplica a matriz de insumos e produtos; $N_{1}$ é o vetor unitário; $N, M$ e $K$ são o número de DMUs, produtos e insumos, respectivamente; e ${ }^{\theta}$ é o escore de eficiência da DMU sob análise.

Essa abordagem forma uma superfície convexa de planos em interseção, a qual envolve os dados de forma mais compacta do que a superfície formada pelo modelo RCE. Com isso, os escores de eficiência técnica são sempre superiores ou iguais àqueles obtidos com o modelo RCE (COELLI, 1996).

Resolvendo o problema de programação linear dos dois modelos RVE e RCE ${ }^{6}$, pode-se decompor a eficiência técnica em duas: pura e de escala. Segundo Abreu et al. (2006), se houver diferença entre os dois escores de eficiência $\left({ }^{\theta} R V E\right.$ e $\left.{ }^{\theta} R C E\right)$ para uma DMU em particular, a DMU possuirá ineficiência de escala e o valor da ineficiência será a razão entre os escores encontrados nos modelos RCE e RVE. A eficiência técnica pura é dada pelo escore de eficiência do modelo RVE.

A ideia do estabelecimento de metas vem do fato de que, em geral, as DMUs não dispõem da informação sobre quanto de produto deveriam ter obtido para que fossem consideradas eficientes. Deveriam, é claro, ter feito o melhor possível com seus recursos. Nesse sentido, uma meta numérica é de grande valia na concepção de melhores diretrizes para os seus esforços (KOZYREFF FILHO; MILIONI, 2004). Contudo, atingir

\footnotetext{
${ }^{5}$ Para maior aprofundamento da discussão, consultar Charnes et al. (1978).

${ }^{6}$ O modelo RCE pode ser representado pela notação algébrica do modelo RVE, desde que se exclua a restrição , que representa o pressuposto da convexidade (COELLI, 1996).
} 
os alvos (metas) determinados pelos modelos DEA é uma tarefa que pode encontrar barreiras práticas. Determinada DMU que busca eficiência pode não ser capaz de alcançar aquele alvo que lhe é atribuído.

O estabelecimento das camadas de eficiência (denominadas camadas de isoeficiência) é, a priori, um artifício para prover a classificação de DMUs em DEA (MELLO et al., 2006), mas sua abordagem para a busca de alvos de forma sequencial foi proposta por Gomes et al. (2003). A partir das sequências, pode-se supor que uma DMU é capaz de promover mudanças em suas práticas de gestão de forma gradual, as quais no médio ou no longo prazo podem ajudar a DMU a atingir patamar de eficiência maior. As vantagens dessa abordagem residem no fato de possibilitar à unidade aprender com o processo e incorporar as mudanças às práticas de gestão para a melhoria do nível de utilização da tecnologia disponível. Ou seja, a unidade pode obter metas em curto prazo mais realistas. Os alvos intermediários, atingidos em sequência, estão localizados nas camadas de isoeficiência, portanto abaixo ou sobre a fronteira eficiente.

Dessa forma, estabelecer meta para uma DMU significa dizer em que proporção uma DMU ineficiente deve reduzir a utilização de seus insumos para que ela seja considerada eficiente. Ou seja, essa meta, se atingida, deve conferir à DMU o status de eficiente. Contudo, cabe ressaltar que, em DEA, ser eficiente não significa que uma unidade não possa melhorar, significa apenas que as unidades ditas eficientes são as que melhor transformam insumos em produtos no grupo analisado.

\subsection{Fonte de dados e procedimentos}

Os dados do Setor de Fabricação de Produtos de Base Florestal (SFPBF) utilizados neste trabalho tiveram como fonte o Instituto Brasileiro de Geografia e Estatística (IBGE) e foram retirados da Pesquisa Industrial Anual (PIA) de 2007, que se encontra disponível em IBGE (2007).

As variáveis selecionadas para a operacionalização do modelo foram:

- Receita Líquida de Vendas (RLV).

- Total de Custos das Operações Industriais (COI).

- Salários, Retiradas e Outras Remunerações (SRO).
A RLV é o produto na DEA e correspondente à diferença entre a receita total e as deduções, sendo uma proxy do resultado do setor. COI e SRO são os insumos. COI representa os custos ligados diretamente à produção industrial (salvo as remunerações) e SRO, as remunerações, sendo usadas como proxys da mão de obra direta e indireta utilizada no setor.

A COI, como definido pelo IBGE (2007), é o resultado da soma do consumo de matérias-primas, materiais auxiliares e componentes; da compra de energia elétrica; do consumo de combustíveis, peças e acessórios; e dos serviços industriais e de manutenção e reparação de máquinas e equipamentos ligados à produção prestados por terceiros. Já a SRO são as importâncias pagas no ano, a título de salários fixos, pró-labore, retiradas de sócios e proprietários, honorários, comissões sobre vendas, ajuda de custo, décimo terceiro salário, abono de férias, gratificações e participação nos lucros.

Como em Anjos (2005), com o objetivo de tornar as DMUs mais homogêneas, optou-se por não incluir na análise as unidades federativas onde o SFPBF era inexpressivo. Assim, analisaram-se as unidades federativas nas quais se localizavam empresas, no ano de 2007, que representassem pelo menos $1 \%$ do total de organizações do SFPBF no país. O critério resultou na incorporação de 95,45\% das empresas pesquisadas pela PIA. Além disso, os Estados selecionados são os mais representativos em termos de pessoal ocupado, valor da transformação industrial e receita total de vendas no ano de 2007.

Os Estados que tiveram a eficiência técnica de seu SFPBF avaliado foram: Rondônia, Pará, Ceará, Pernambuco, Bahia, Minas Gerais, Espírito Santo, Rio de Janeiro, São Paulo, Paraná, Santa Catarina, Rio Grande do Sul, Mato Grosso e Goiás. Na DEA, cada um desses Estados representa uma DMU.

Utilizou-se o modelo DEA-RVE, posto que as unidades avaliadas apresentavam diferenças de escala significativas e, segundo Coelli (1996), o uso do modelo DEA-RVE permite o cálculo da eficiência técnica isenta dos efeitos da eficiência de escala. Ademais, optou-se pela orientação ao insumo, uma vez que a proposição de aumento expressivo do total da receita líquida de todo um setor industrial é irreal.

Revista Árvore, Viçosa-MG, v.35, n.6, p.1319-1326, 2011 
As camadas de isoeficiência para o estabelecimento das metas de curto prazo foram obtidas como indicado por Gomes et al. (2003): as DMUs com $100 \%$ de eficiência formaram a camada 1. Essas DMUs foram, então, retiradas do conjunto de análise, e procedeu-se novamente à análise. As DMUs eficientes nesse subconjunto formaram a camada 2. O processo repetiu-se até que todas as DMUs tivessem sido retiradas do conjunto inicial.

A eficiência de escala foi analisada pelos escores dos modelos RVE ( $\left.{ }^{\theta} R V E\right)$ e RCE $\left({ }^{\theta} R C E\right)$. Quando se obteve o mesmo escore nos dois modelos, foi determinada a DMU que apresentava eficiência de escala, operando com retornos constantes à escala; caso contrário, a DMU exibia ineficiência de escala, que foi calculada pela razão entre ${ }^{\theta} R C E \mathrm{e}^{\theta} R V E$. O tipo de retorno à escala apresentado pelas DMUs ineficientes foi determinado comparando os escores do modelo RVE e $\mathrm{NDE}^{7}$ (Retornos Não Decrescentes à escala: se ${ }^{\theta} R V E \neq{ }^{\theta} R C E$, a DMU operava com retornos crescentes à escala; se , operava com retornos decrescentes à escala.

\section{RESULTADOS}

Para obter os alvos de curto prazo das DMUs ineficientes, geraram-se seis camadas de isoeficiência. Dos 14 Estados analisados, Espírito Santo e Rio Grande do Sul foram considerados eficientes, compondo a camada 1 de isoeficiência (Tabela 1).

Era esperado que, devido à concorrência, o Estado que apresentasse o maior número de firmas no SFPBF tivesse maior eficiência em relação aos demais, porém o Estado do Espírito Santo, com 246 firmas (1,75\% do total de firmas), mostrou possuir maior eficiência no setor industrial analisado ${ }^{8}$. Portanto, a maior eficiência do Espírito Santo é justificável. Esse Estado, segundo Valverde et al. (2005), possui condições naturais favoráveis, aliadas ao desenvolvimento tecnológico avançado da silvicultura e a outras condições privilegiadas, como localização geográfica, infraestrutura, logística de transporte, diversificação de plantas industriais etc., constituindo-se de elementos que alavancam o potencial de crescimento da atividade florestal. Tais elementos permitem estrutura de gastos

Tabela 1 - Redução (\%) necessária nos custos para que cada Estado atinja uma fronteira de isoeficiência superior em 2007. Table 1 - Necessary reduction (\%) in costs for each state reaches a superior frontier of isoefficiency in 2007.

\begin{tabular}{|c|c|c|c|c|c|c|c|c|c|c|}
\hline \multirow{3}{*}{$\begin{array}{l}\text { Unidade } \\
\text { Federativa }\end{array}$} & \multicolumn{8}{|c|}{ Fronteira de Isoeficiência } & \multirow{2}{*}{\multicolumn{2}{|c|}{$5 *$}} \\
\hline & \multicolumn{2}{|c|}{1} & \multicolumn{2}{|c|}{2} & \multicolumn{2}{|c|}{3} & \multicolumn{2}{|c|}{4} & & \\
\hline & COI & $\overline{\text { SRO }}$ & COI & $\overline{\mathrm{SRO}}$ & COI & SRO & COI & $\overline{\mathrm{SRO}}$ & COI & $\overline{\text { SRO }}$ \\
\hline $\mathrm{RO}$ & 7.29 & 7.29 & 0 & 0 & - & - & - & - & - & - \\
\hline $\mathrm{PA}$ & 33.33 & 33.33 & 16.67 & 16.67 & 2.78 & 2.78 & 0 & 0 & - & - \\
\hline $\mathrm{CE}$ & 47.71 & 29.94 & 29.44 & 21.74 & 14.45 & 14.45 & 12.89 & 12.89 & 0 & 0 \\
\hline $\mathrm{PE}$ & 42.72 & 32.28 & 23.12 & 23.12 & 9.4 & 9.4 & 7.47 & 7.47 & 0 & 0 \\
\hline BA & 9.49 & 19.251 & 21.74 & 0.49 & 0 & 0 & - & - & - & - \\
\hline MG & 25.9 & 10.48 & 0 & 0 & - & - & - & - & - & - \\
\hline ES & 0 & 0 & - & - & - & - & - & - & - & - \\
\hline $\mathrm{RJ}$ & 46.02 & 46.02 & 30.95 & 30.95 & 19.14 & 19.14 & 17.05 & 17.05 & 16.07 & 16.07 \\
\hline SP & 6.10 & 6.10 & 0 & 0 & - & - & - & - & - & - \\
\hline PR & 40.3 & 12.14 & 19.43 & 1.85 & 0 & 0 & - & - & - & - \\
\hline SC & 35.1 & 35.1 & 17.26 & 17.26 & 3.17 & 3.17 & 0.62 & 0.62 & 0 & 0 \\
\hline RS & 0 & 0 & - & - & - & - & - & - & - & - \\
\hline MT & 25.74 & 25.74 & 13.37 & 13.37 & 0 & 0 & - & - & - & - \\
\hline GO & 40.1 & 19.1 & 19.15 & 9.62 & 1.78 & 1.78 & 0 & 0 & - & - \\
\hline
\end{tabular}

Fonte: Elaboração do autor.

* A última camada (Rio de Janeiro).

${ }^{7} \mathrm{O}$ modelo DEA-NDE pode ser representado pela notação algébrica do modelo RVE, desde que se substitua a restrição $N{ }_{1} \lambda=1$ pela $N^{\prime}{ }_{1} \lambda \geq 1$ (COELLI, 1996).

${ }^{8}$ Classificou-se São Paulo como a DMU mais eficiente entre as que compõem a camada 1 de isoeficiência pelo índice de supereficiência. É o estado que pode sofrer maiores aumentos em seus insumos sem deixar de ser eficiente pela camada $1 .$. 
mais equilibrada, propiciando menores custos operacionais (COI), que foram os principais geradores de ineficiência nos Estados analisados.

O Estado do Espírito Santo também foi o que apresentou as melhores condições custo/benefício, pois o COI significava 35,75\% da RLV e o SRO, 7,67\%. No segundo Estado mais eficiente (Rio Grande do Sul), o COI representava $54,97 \%$ e o SRO, $12,18 \%$.

Entre os insumos utilizados na análise, nos Estados do Espírito Santo e Rio Grande do Sul o COI teve, respectivamente, pesos 0,72 e 1 , mostrando-se o principal responsável pela eficiência dessas DMUs. Ou seja, esses Estados foram eficientes porque apresentaram baixo custo nas operações industriais em relação à RLV, e essa foi a tendência verificada em todos os Estados analisados na primeira estimativa do SFPBF.

As DMUs ineficientes pela camada 1 de isoeficiência obtiveram escore médio de 77,75\%. Entretanto, as DMUs que mais se aproximam da camada de maior eficiência foram, evidentemente, aquelas que compõem a camada 2 de isoeficiência (Tabela 1): Rondônia, Minas Gerais e São Paulo. Na Tabela 1, mostra-se quanto essas DMUs devem reduzir o SRO e o COI para atingir a primeira camada, considerando as folgas nos insumos. Sugere-se que essas reduções sejam tidas como metas de curto prazo para essas DMUs, posto que o Estado de São Paulo, por exemplo, deveria reduzir SRO e COI em apenas 6,1\% para ser considerado eficiente, e Minas Gerais deveria diminuir SRO em 10,48\% e COI em 25,9\% para atingir a camada 1 , sem folga nos insumos.
Pela camada 2, identificam-se nove DMUs ineficientes (Tabela 1), com um escore médio de 84,99\%. Os Estados que devem buscar atingir a camada 2 no curto prazo são Pará, Ceará, Pernambuco, Rio de Janeiro, Santa Catarina e Goiás. Rio de Janeiro é o Estado que deve fazer o maior esforço para atingir a camada 2 com redução geral dos custos de 30,95\%. Na camada 3, Bahia, Paraná e Mato Grosso foram os mais eficientes, seguidos na camada 4 por Pará e Goiás. Na camada 5, dos quatro Estados restantes, três foram eficientes (Ceará, Pernambuco e Santa Catarina), ficando apenas o Rio de Janeiro na última camada. Isso evidencia que, dos Estados analisados, o Rio de Janeiro tem o pior desempenho em eficiência e o que mais precisará fazer ajustes para atingir maiores patamares de eficiência.

\section{DISCUSSÃO}

Na Tabela 2, verifica-se que aqueles Estados com maior número de empresas no SFPBF, onde se espera que esse setor seja mais desenvolvido, operam com retornos decrescentes à escala.

Num mercado competitivo, supondo-se uma curva de custo médio de longo prazo em forma de $\mathbf{U}$, a otimização do lucro só é compatível com retornos decrescentes à escala ou no limiar do ramo da curva de custo médio de longo prazo que apresenta retornos decrescentes à escala (ALVES, 2004). Entretanto, o SFPBF não se caracteriza como mercado perfeitamente competitivo ${ }^{9}$, pois há grande rivalidade entre as firmas, levando-as a apresentar diferenças nos produtos oferecidos.

Tabela 2 - Número de empresas, eficiência de escala e tipos de retornos à escala por Estado, em 2007. Table 2 - Number of firms, efficiency of scale and types of returns to scale by state in 2007.

\begin{tabular}{cccc}
\hline Unidade Federativa & $\mathrm{N}^{\circ}$ de Empresas & Eficiência de Escala (\%) & Tipo de retorno \\
\hline RO & 428 & 92.71 & Crescente \\
PA & 802 & 66.67 & Decrescente \\
CE & 143 & 70.06 & Crescente \\
PE & 145 & 67.72 & Crescente \\
BA & 249 & 90.51 & Crescente \\
MG & 786 & 89.52 & Decrescente \\
ES & 246 & 100 & Constante \\
RJ & 358 & 53.98 & Crescente \\
SP & 5202 & 93.9 & Decrescente \\
PR & 1758 & 87.86 & Decrescente \\
SC & 1961 & 64.9 & Decrescente \\
RS & 1083 & 100 & Constante \\
MT & 722 & 74.26 & Crescente \\
GO & 142 & 80.9 & Crescente \\
\hline
\end{tabular}

Fonte: Elaborado do autor.

Revista Árvore, Viçosa-MG, v.35, n.6, p.1319-1326, 2011 
De acordo com Losekann e Gutierrez (2002), a diferenciação é fator que gera barreiras à entrada, já que as empresas entrantes têm de realizar gastos substanciais com esforços de venda para reverter a preferência dos consumidores por produtos de firmas já estabelecidas. Assim, espera-se que as barreiras geradas pela diferenciação dos produtos do SFPBF estabeleçam dificuldade à expansão desse setor pela entrada de novas empresas, fazendo com que o SFPBF seja, provavelmente, otimizado com retornos decrescentes à escala. Corroborando essa hipótese, encontraram-se retornos crescentes à escala nos Estados onde o SFPBF ainda é incipiente e retornos decrescentes à escala naquelas unidades federativas onde esse setor é mais desenvolvido, com grande número de empresas já estabelecidas (Tabela 2).

Das 14DMUs analisadas, nove operavam com retornos constantes ou crescentes à escala, com três dessas DMUs estando muito próximas de 100\% de eficiência. Logo, esses estados devem tentar romper as barreiras à expansão para atingir o ramo da curva de custo médio de longo prazo com retornos decrescentes à escala, especialmente São Paulo e Rondônia (Tabela 2), que podem obter ganhos expressivos no SFPBF com a expansão da produção com a atual estrutura produtiva, pois com retornos crescentes à escala essa expansão leva a RLV a crescer mais que os custos SRO e COI.

\section{CONCLUSÃO}

A análise da eficiência técnica indica que, em geral, a principal fonte de ineficiência dos Estados analisados são os altos custos das operações industriais (COI), sinalizando aos empresários do SFPBF de cada Estado a necessidade de um controle austero dos fatores que compõem seus custos, devendo direcionar maiores esforços para a redução, principalmente, do COI. Uma forma de diminuir tais custos seria pela iniciativa pública em baixar a carga tributária, que, segundo empresários de todos os setores de transformação, onera e impede o avanço de novos investimentos no país.

O uso da DEA para o estabelecimento de metas de curto prazo proporciona, portanto, informações importantes sobre quanto as DMUs devem reduzir seus custos, através de alvos intermediários, factíveis, no curto prazo, para se tornarem tecnicamente eficientes no grupo analisado, e quais custos são os principais responsáveis pela sua ineficiência. No médio prazo, os Estados de Rondônia, São Paulo e Minas Gerais devem fazer o esforço de adequação de seus custos operacionais e salários para alcançar a camada 1 (à qual pertencem Espírito Santo e Rio Grande do Sul).

A análise da eficiência de escala apontou que os Estados onde o SFPBF é mais desenvolvido operam com retornos decrescentes à escala em razão, possivelmente, das barreiras à entrada geradas pela diferenciação dos produtos do setor. Verificou-se que a maioria dos Estados operava com retornos constantes ou crescentes, razão por que devem expandir o SFPBF dada a possibilidade de ganhos expressivos, especialmente para Rondônia e São Paulo.

\section{REFERÊNCIAS}

ABREU, U. G. P. et al. Avaliação da introdução de tecnologias no sistema de produção de gado de corte no Pantanal: Análise de eficiência. Revista Brasileira de Zootecnia, v.35, n.3, p.1242-1250, 2006.

ALVES, E. Retornos à escala e mercado competitivo: teoria e evidências empíricas.

Revista de Economia e Agronegócio, v.2, n.3, p.311-334, 2004.

ANJOS, M. A. Aplicação da análise envoltória de dados no estudo da eficiência econômica da indústria têxtil brasileira nos anos 90. 2005. 239f. Tese (Doutorado em Engenharia de Produção) Centro Tecnológico, Universidade Federal de Santa Catarina, Florianópolis, 2005.

BECKER, J. L.; LUNARDI, G. L.; MAÇADA, A. C. G. Análise de eficiência dos bancos brasileiros: um enfoque nos investimento realizados em Tecnologia de Informação (TI). Revista Produção, v.13, n.2, p.70-81, 2003.

CHARNES, A.; COOPER, W. W.; RHODES, E. Measuring the efficiency of decision-making units. European Journal of Operational Research, v.2, n.6, p.429-444l, 1978.

\footnotetext{
${ }^{9}$ Um mercado perfeitamente competitivo é inteiramente impessoal, não havendo nenhum traço de rivalidade entre as firmas (FERGUSON, 2003).
} 
COELLI, T. J. A Guide to DEAP Version 2.1: A Data Envelopment Analysis (Computer) Program. Centre for Efficiency and Productivity Analysis - CEPA 1996. 50p. (Working Paper, 8)

DULÁ, J. H. Computations in DEA. Pesquisa Operacional, v.22, n.2, p.165-182, 2002.

FERGUSON, C. E. Microeconomia. 20.ed. Rio de Janeiro: Forense-Universitária, 2003.

GOMES, A. P.; BAPTISTA, A. J. M. S. Análise envoltória de dados: conceitos e modelos básicos. In: SANTOS, M. L.; VIEIRA, W. C. (Eds.). Métodos quantitativos em economia. Viçosa, MG: Universidade Federal de Viçosa, 2004. p.121-160.

GOMES, E. G.; MANGABEIRA, J. A. C.; MELLO, J. C. C. B. S. Análise envoltória de dados para avaliação de eficiência e caracterização de tipologias em agricultura: um estudo de caso.

Revista de Economia Sociologia Rural, v.43, n.4, p.607-631, 2005.

GOMES, E. G.; MELLO, J. C.; LINS, M. P. Busca seqüencial de alvos intermediários em modelos DEA com soma de outputs constante.

Investigação Operacional, v.23, n.2, p.163-178, 2003.

\section{INSTITUTO BRASILEIRO DE GEOGRAFIA E} ESTATISTICA - IBGE. Pesquisa

Industrial Anual (PIA). Brasil, 2007. Disponível em: < http://www.sidra.ibge.gov.br/ bda/pesquisas/pia/default.asp $\mathrm{o}=16 \& \mathrm{i}=\mathrm{P}>$. Acesso em: 23 de abr. de 2010.
KOZYREFF FILHO, E.; MILIONI, A. Z. Um método para estimativa de metas DEA. Revista Produção, v.14, n.2, p.70-81, 2004.

LETA, F. R. et al. Métodos de melhora de ordenação em DEA aplicados à avaliação estática de tornos mecânicos. Investigação Operacional, v.25, n.2, p.229-242, 2005.

LOSEKANN, L.; GUTIERREZ, M. Diferenciação de produtos. In: KUPFER, D.; HASENCLEVER, L. (Org.). Economia Industrial. Fundamentos teóricos e práticas no Brasil. Rio de Janeiro: Elsevier, 2002. p.91-128.

MELLO, J. C. C. B. S.; MEZA, L. A.; GOMES, E. G. Eficiência no consumo de energia em municípios fluminenses considerando temperaturas. Niterói: 2006. 8p. (Relatórios de Pesquisa em Engenharia de Produção).

SAMPAIO, L. M. B.; RAMOS, F. S.; SAMPAIO, Y. Privatização e eficiência das usinas hidrelétricas brasileiras. Economia Aplicada, v.9, n.3, p.465-480, 2005.

SANTANA, A. C. A competitividade sistêmica das empresas de madeira da região Norte. Belém: Fundação Biblioteca Nacional, 2002.

SIMONSEN, M. H. Teoria microeconômica. 11.ed. Rio de Janeiro: Fundação Getúlio Vargas, 1993. v.1.

VALVERDE, S. R. et al. Participação do setor florestal nos indicadores socioeconômicos do estado do Espírito Santo. Revista Árvore, v.29, n.1, p.105-113, 2005.

VARIAN, H. R. Microeconomia: princípios básicos. Rio de Janeiro: Campus, 2000. 